\title{
Rings of symmetric functions as modules over the Steenrod algebra
}

\author{
WILLIAM M SINGER
}

\begin{abstract}
We write $P^{\otimes s}$ for the polynomial ring on $s$ letters over the field $\mathbb{Z} / 2$, equipped with the standard action of $\Sigma_{s}$, the symmetric group on $s$ letters. This paper deals with the problem of determining a minimal set of generators for the invariant ring $\left(P^{\otimes s}\right)^{\Sigma_{s}}$ as a module over the Steenrod algebra $\mathcal{A}$. That is, we would like to determine the graded vector spaces $\mathbb{Z} / 2 \otimes_{\mathcal{A}}\left(P^{\otimes s}\right)^{\Sigma_{s}}$. Our main result is stated in terms of a "bigraded Steenrod algebra" $\mathcal{H}$. The generators of this algebra $\mathcal{H}$, like the generators of the classical Steenrod algebra $\mathcal{A}$, satisfy the Adem relations in their usual form. However, the Adem relations for the bigraded Steenrod algebra are interpreted so that $\mathbb{S} q^{0}$ is not the unit of the algebra; but rather, an independent generator. Our main work is to assemble the duals of the vector spaces $\mathbb{Z} / 2 \otimes_{\mathcal{A}}\left(P^{\otimes s}\right)^{\Sigma_{s}}$, for all $s \geq 0$, into a single bigraded vector space and to show that this bigraded object has the structure of an algebra over $\mathcal{H}$.
\end{abstract}

13A50, 55S10; 18G15, 55Q45, 55T15, 18G10

\section{Introduction}

We write $P$ for the polynomial ring in one variable over the finite field $\mathbb{Z} / 2$ and $P^{\otimes s}=\mathbb{Z} / 2\left[t_{1}, \ldots t_{s}\right]$ for its $s$-fold tensor product with itself. We grade $P^{\otimes s}$ by assigning degree 1 to each generator. The general linear group $G L_{S}(\mathbb{Z} / 2)$ acts in the usual way on $P^{\otimes s}$ as a group of algebra automorphisms. A central problem of invariant theory is to determine the ring of invariants $\left(P^{\otimes s}\right)^{H_{s}}$ for a given subgroup $H_{S} \subseteq G L_{S}(\mathbb{Z} / 2)$. Because we are working over $\mathbb{Z} / 2$ we are interested in more than just the ring structure of $\left(P^{\otimes s}\right)^{H_{s}}$. We also want to understand the action of the Steenrod algebra $\mathcal{A}$ on the invariant ring. It is well-known how this action comes about. $P^{\otimes s}$ is the $\mathbb{Z} / 2-$ cohomology of the classifying space of the elementary abelian 2-group of rank $s$ :

$$
P^{\otimes s}=H^{*}\left(B(\mathbb{Z} / 2)^{\times s}, \mathbb{Z} / 2\right) .
$$

This representation of $P^{\otimes s}$ defines it as an $\mathcal{A}$-module. The Steenrod operations are natural with respect to maps of topological spaces, so they commute with the action 
of $G L_{s}(\mathbb{Z} / 2)$ on $P^{\otimes s}$. Then for each subgroup $H_{s} \subseteq G L_{s}(\mathbb{Z} / 2)$, the invariant ring $\left(P^{\otimes s}\right)^{H_{s}}$ is stable under the action of $\mathcal{A}$, and becomes an $\mathcal{A}$-module in its own right. Then it is natural to ask: what is a minimal set of generators for $\left(P^{\otimes s}\right)^{H_{s}}$ as an $\mathcal{A}$-module? Equivalently, can one write down a basis for the graded vector space:

$$
\mathbb{Z} / 2 \otimes_{\mathcal{A}}\left(P^{\otimes s}\right)^{H_{s}} .
$$

Even in cases in which $\left(P^{\otimes s}\right)^{H_{s}}$ is well understood as a ring, this problem has proved surprisingly difficult. In the case in which $H_{s}=\{e\}$, the trivial group, the vector space (1-2) is completely known if $s=3$ : see Kameko [10] and Boardman [3]. The case $s=4$ has been treated by Kameko [9] and by Sum [24]; both manuscripts are unpublished at the time of this writing. But when $H_{s}=\{e\}$ and $s \geq 5$, the vector space (1-2) is not yet known. Approaches to the problem when $H_{S}=\{e\}$, for general $s$, can be found in the work of Alghamdi-Crabbe-Hubbuck [2], Crabbe-Hubbuck [5], Carlyle-Wood [4], Meyer-Silverman [13], Nam [15], Peterson [16], Silverman [17], Silverman-Singer [18], Wood [25;26] and the present writer [20]. Applications to homotopy theory are discussed by Peterson [16], and by the present writer [19]. For the case in which $H_{s}=G L_{s}(\mathbb{Z} / 2)$ the vector space (1-2) is known if $s=2$ [19], and if $s=3,4$ from the work of Hung-Peterson [6].

The present paper is a study of the graded vector spaces:

$$
\mathbb{Z} / 2 \otimes_{\mathcal{A}}\left(P^{\otimes s}\right)^{\Sigma_{s}}, \quad s \geq 0,
$$

where $\Sigma_{s}$ is the symmetric group on $s$ letters. Work on this problem was begun by Janfada and Wood in [7; 8]. In particular these authors find a basis for $\mathbb{Z} / 2 \otimes_{\mathcal{A}}\left(P^{\otimes 3}\right)^{\Sigma_{3}}$. In the present work we assemble the duals of the graded vector spaces (1-3) for all $s \geq 0$, into a single, bigraded vector space. Our main result, given formally in Theorem 1.3 and Theorem 1.4, is that this bigraded vector space can be given the structure of an algebra over a "bigraded Steenrod algebra".

In order to state our main result precisely we need to define the "bigraded Steenrod algebra", and distinguish it from the classical one. The bigraded Steenrod algebra $\mathcal{H}$ is generated by symbols $\left\{\mathbb{S} q^{l} \mid l \geq 0\right\}$, and is subject to Adem relations as they are usually written:

$$
\mathbb{S} q^{a} \mathbb{S} q^{b}=\sum_{j \geq 0}\left(\begin{array}{c}
b-1-j \\
a-2 j
\end{array}\right) \mathbb{S} q^{a+b-j} \mathbb{S} q^{j} ; \quad 0 \leq a<2 b .
$$

In interpreting these relations we understand that $\mathbb{S} q^{0}$ is a generator on the same footing as the others; so, for example, $\mathbb{S} q^{1} \mathbb{S} q^{2}=\mathbb{S} q^{3} \mathbb{S} q^{0}$. Because the relations (1-4) are homogeneous of length 2 in the generators, $\mathcal{H}$ is graded not only by the 
superscripts of the Steenrod squares, but also by the length of a monomial in the generators: $\operatorname{deg}\left(\mathbb{S} q^{l}\right)=(l, 1)$. This bigraded Steenrod algebra was introduced by Liulevicius in [11]. Its properties were developed further by May [12] and by the present writer [21].

The classical Steenrod algebra $\mathcal{A}$ is generated by symbols $\left\{S q^{l} \mid l \geq 0\right\}$ and subject to the Adem relations:

$$
S q^{a} S q^{b}=\sum_{j \geq 0}\left(\begin{array}{c}
b-1-j \\
a-2 j
\end{array}\right) S q^{a+b-j} S q^{j}, \quad 0 \leq a<2 b,
$$

as well as the important relation $S q^{0}=1$. For example, $S q^{1} S q^{2}=S q^{3} . \mathcal{A}$ is a singly graded algebra, with the grading determined by $\operatorname{deg}\left(S q^{l}\right)=l$. Steenrod and Epstein [23] is a standard reference for the properties of $\mathcal{A}$.

We make the following definition.

Definition 1.1 Let $\mathcal{H}$ be the bigraded Steenrod algebra, as defined above. By an $\mathcal{H}$-algebra we mean a bigraded $\mathbb{Z} / 2$-algebra $\Delta$ that is also a left $\mathcal{H}$-module, in such a way that:

(1) the generators $\mathbb{S} q^{l}$ treat the bigrading on $\Delta$ according to the formula:

$$
\mathrm{S} q^{l}: \Delta_{s, t} \rightarrow \Delta_{s+l, 2 t}
$$

(2) the action of $\mathcal{H}$ on $\Delta$, and the product on $\Delta$, are related by the Cartan formula:

$$
\mathbb{S} q^{l}(\alpha \beta)=\sum_{i+j=l}\left(\mathbb{S} q^{i} \alpha\right)\left(\mathbb{S} q^{j} \beta\right)
$$

(3) if $\alpha \in \Delta_{s^{\prime}, *}$ then:

$$
\mathbb{S} q^{s} \alpha=\left\{\begin{array}{lll}
\alpha^{2} & \text { if } & s=s^{\prime} \\
0 & \text { if } & s>s^{\prime}
\end{array}\right.
$$

In Section 2 we will recall the context in which Liulevicius introduced his Steenrod operations, thereby providing some motivation for the above definition.

In stating our results it is convenient to work with the duals of the vector spaces (1-1) and (1-3). So we define a bigraded vector space $\Gamma=\left\{\Gamma_{s, t} \mid s \geq 0, t \geq 0\right\}$ by writing:

$$
\Gamma_{s, t}=H_{t-s}\left(B(\mathbb{Z} / 2)^{\times s}, \mathbb{Z} / 2\right) .
$$

(In particular, $\Gamma_{0, *}$ is the homology of a point.) Motivation for this choice of grading will be given in Section 2. For each $k \geq 0$ we will write

$$
\gamma_{k} \in \Gamma_{1, k+1}=H_{k}(B(\mathbb{Z} / 2), \mathbb{Z} / 2)
$$


for the canonical generator. The cross product:

$(1-10) \quad H_{*}\left(B(\mathbb{Z} / 2)^{\times s}, \mathbb{Z} / 2\right) \otimes H_{*}\left(B(\mathbb{Z} / 2)^{\times s^{\prime}}, \mathbb{Z} / 2\right) \rightarrow H_{*}\left(B(\mathbb{Z} / 2)^{\times\left(s+s^{\prime}\right)}, \mathbb{Z} / 2\right)$

makes $\Gamma$ into a connected, bigraded algebra. We will write $\alpha \times \beta$ for the image of $\alpha \otimes \beta$ under this pairing. For each $s \geq 0$ the vector space $\Gamma_{s, *}$ is the dual of the vector space $P^{\otimes s}$, except for the shift in grading. So $\Gamma_{s, *}$ acquires an $\mathcal{A}$-action that is dual to the $\mathcal{A}$-action on $P^{\otimes s}$. This action is from the right and lowers internal degree. As is well known we have the Cartan formula for Steenrod operations on cross products:

$$
(\alpha \times \beta) S q^{l}=\sum_{i+j=l} \alpha S q^{i} \times \beta S q^{j} .
$$

We will write $\left(\Gamma_{s, *}\right)^{\mathcal{A}}$ for the " $\mathcal{A}$-annihilated" elements of $\Gamma_{s, *}$ :

$$
\left(\Gamma_{s, *}\right)^{\mathcal{A}}=\left\{\forall \alpha \in \Gamma_{s, *} \mid(\alpha) S q^{l}=0, \forall l>0\right\} .
$$

We assemble these graded vector spaces into a single, bigraded vector space $\Gamma^{\mathcal{A}}$ by writing $\left(\Gamma^{\mathcal{A}}\right)_{s, *}=\left(\Gamma_{s, *}\right)^{\mathcal{A}}$. The Cartan formula $(1-11)$ implies that $\Gamma^{\mathcal{A}}$ is a subalgebra of $\Gamma$.

We define a bigraded vector space $\mathbb{Z} / 2 \otimes_{\Sigma} \Gamma$ by writing:

$$
\left(\mathbb{Z} / 2 \otimes_{\Sigma} \Gamma\right)_{s, *}=\mathbb{Z} / 2 \otimes_{\Sigma_{s}} \Gamma_{s, *}, \quad \text { all } s \geq 0 .
$$

As above, $\gamma_{k} \in\left(\mathbb{Z} / 2 \otimes_{\Sigma} \Gamma\right)_{1, k+1}$ is the generator, for each $k \geq 0$. It is easy to see that $\mathbb{Z} / 2 \otimes_{\Sigma} \Gamma$ is a commutative quotient algebra of $\Gamma$. We will write $\alpha \beta$ for the product of elements $\alpha, \beta \in \mathbb{Z} / 2 \otimes_{\Sigma} \Gamma$. For each $s \geq 0$ the vector space $\left(\mathbb{Z} / 2 \otimes_{\Sigma} \Gamma\right)_{s, *}$ is the dual of the vector space $\left(P^{\otimes s}\right)^{\Sigma_{s}}$, except for the shift in grading. So $\left(\mathbb{Z} / 2 \otimes_{\Sigma} \Gamma\right)_{s, *}$ acquires an $\mathcal{A}$-action that is dual to the $\mathcal{A}$-action on $\left(P^{\otimes s}\right)^{\Sigma_{s}}$. This action is clearly the same as that obtained from the action of $\mathcal{A}$ on $\Gamma_{s, *}$ by passage to the quotient. Consequently we have from (1-11) a Cartan formula for the $\mathcal{A}$-action on $\mathbb{Z} / 2 \otimes_{\Sigma} \Gamma$ :

$$
(\alpha \beta) S q^{l}=\sum_{i+j=l}\left(\alpha S q^{i}\right)\left(\beta S q^{j}\right) .
$$

Finally we write $\left(\mathbb{Z} / 2 \otimes_{\Sigma} \Gamma\right)^{\mathcal{A}}$ for the bigraded vector space defined by:

$$
\left(\left(\mathbb{Z} / 2 \otimes_{\Sigma} \Gamma\right)^{\mathcal{A}}\right)_{s, *}=\left(\left(\mathbb{Z} / 2 \otimes_{\Sigma} \Gamma\right)_{s, *}\right)^{\mathcal{A}}, \quad \text { all } s \geq 0 .
$$

Here the right-hand side refers to the $\mathcal{A}$-annihilated elements of $\left(\mathbb{Z} / 2 \otimes_{\Sigma} \Gamma\right)_{s, *}$, as in (1-12). The Cartan formula (1-14) implies that $\left(\mathbb{Z} / 2 \otimes_{\Sigma} \Gamma\right)^{\mathcal{A}}$ is a subalgebra of $\mathbb{Z} / 2 \otimes_{\Sigma} \Gamma$. 
Remark 1.2 For each $s \geq 0,\left(\left(\mathbb{Z} / 2 \otimes_{\Sigma} \Gamma\right)^{\mathcal{A}}\right)_{s, *}$ is the dual of the vector space (1-3), except for our shift in the grading. Thus, the determination of $\left(\left(\mathbb{Z} / 2 \otimes_{\Sigma} \Gamma\right)^{\mathcal{A}}\right)_{s, *}$ would be equivalent to finding a minimal set of generators for $P^{\otimes s}$ as an $\mathcal{A}$-module.

The bigraded algebra $\left(\mathbb{Z} / 2 \otimes_{\Sigma} \Gamma\right)^{\mathcal{A}}$ is the main subject of our paper.

We can now state our two theorems.

Theorem 1.3 There exists a left action of the bigraded Steenrod algebra $\mathcal{H}$ on the bigraded algebra $\mathbb{Z} / 2 \otimes_{\Sigma} \Gamma$, such that

$$
\mathbb{S} q^{0} \gamma_{k}=\gamma_{2 k+1}, \quad \text { all } k \geq 0,
$$

and such that $\mathbb{Z} / 2 \otimes_{\Sigma} \Gamma$ becomes an $\mathcal{H}$-algebra in the sense of Definition 1.1. The action of $\mathcal{H}$ on $\mathbb{Z} / 2 \otimes_{\Sigma} \Gamma$ that satisfies these conditions is unique.

Theorem 1.4 The subalgebra $\left(\mathbb{Z} / 2 \otimes_{\Sigma} \Gamma\right)^{\mathcal{A}} \subseteq \mathbb{Z} / 2 \otimes_{\Sigma} \Gamma$ is stable under the action of $\mathcal{H}$ that is described in Theorem 1.3. Therefore $\left(\mathbb{Z} / 2 \otimes_{\Sigma} \Gamma\right)^{\mathcal{A}}$ itself becomes an $\mathcal{H}$-algebra.

We remark that the vector spaces $\left(\mathbb{Z} / 2 \otimes_{\Sigma} \Gamma\right)_{s, *}$ have a topological interpretation. It is well known that the cohomology of the classifying space of the orthogonal group is the invariant ring: $H^{*}(B O(s), \mathbb{Z} / 2)=\left(P^{\otimes s}\right)^{\Sigma_{s}}$. Consequently:

$$
\left(\mathbb{Z} / 2 \otimes_{\Sigma} \Gamma\right)_{s, t}=H_{t-s}(B O(s), \mathbb{Z} / 2), \quad \forall s, t \geq 0 .
$$

Our statement that $\mathbb{Z} / 2 \otimes_{\Sigma} \Gamma$ can be given the structure of an $\mathcal{H}$-algebra says in particular that the Steenrod squares treat bidegree according to the rule:

$$
\mathbb{S} q^{l}:\left(\mathbb{Z} / 2 \otimes_{\Sigma} \Gamma\right)_{s, t} \rightarrow\left(\mathbb{Z} / 2 \otimes_{\Sigma} \Gamma\right)_{s+l, 2 t},
$$

and so: $\quad \quad S q^{l}: H_{n}(B O(s), \mathbb{Z} / 2) \rightarrow H_{2 n+s-l}(B O(s+l), \mathbb{Z} / 2)$.

It would be interesting to have a geometric interpretation of these operations.

The plan of the paper is as follows. In Section 2 we describe earlier constructions of Liulevicius [11], May [12] and the author [19] that motivate our Definition 1.1 and our Theorem 1.3 and Theorem 1.4. In Section 3 we recall from our paper [21] some results on the structure of $\mathcal{H}$. These will turn out to be helpful in the proofs of Theorem 1.3 and Theorem 1.4. In Section 4 we prove Theorem 1.3, and in Section 5 we prove Theorem 1.4. In Section 6 we do some sample computations, showing how the operators $\mathbb{S} q^{l}$ can be applied to low dimensional elements of $\left(\mathbb{Z} / 2 \otimes_{\Sigma} \Gamma\right)^{\mathcal{A}}$ to obtain interesting elements of higher degree. 


\section{Motivation}

The purpose of this section is to describe some earlier work of Liulevicius [11] in which the bigraded Steenrod operations were introduced, some earlier work of May [12] on the structure of the bigraded Steenrod algebra, and some earlier work of the author [19] which related invariant theory to the cohomology of the singly graded Steenrod algebra. From a strictly logical point of view this section is not prerequisite to the rest of the paper. Nevertheless we hope it will explain the provenance of Definition 1.1, explain how the author was led to formulate Theorem 1.3 and Theorem 1.4, and help to place those theorems in a broader context.

We begin by recalling a construction due to Liulevicius [11]. Let $\mathcal{C}$ be any graded, cocommutative Hopf algebra over $\mathbb{Z} / 2$. We write:

$$
\operatorname{Ext}_{\mathcal{C}}(\mathbb{Z} / 2, \mathbb{Z} / 2)=\left\{\operatorname{Ext}_{\mathcal{C}}^{s, t}(\mathbb{Z} / 2, \mathbb{Z} / 2) \mid s \geq 0, t \geq 0\right\}
$$

for the usual bigraded cohomology ring of $\mathcal{C}$. Here $s$ labels the homological degree, and $t$ labels the internal degree that comes from the grading of $\mathcal{C}$. In [11], Liulevicius constructs Steenrod squaring operations:

$$
\mathbb{S}^{l}: \operatorname{Ext}_{\mathcal{C}}^{s, t}(\mathbb{Z} / 2, \mathbb{Z} / 2) \rightarrow \operatorname{Ext}_{\mathcal{C}}^{s+l, 2 t}(\mathbb{Z} / 2, \mathbb{Z} / 2), \quad l \geq 0 .
$$

(Actually Liulevicius is working over odd primes in [11], but the analogous constructions at $p=2$ are easily inferred from his work. They are written out explicitly, for example, in May [12], Milgram [14] and our book [22].) The definition of the operations (2-2) uses "cup- $i$ products" on a projective resolution of $\mathbb{Z} / 2$ as a $\mathcal{C}$-module. In particular one has immediately from the definitions that if $\alpha \in \operatorname{Ext}_{\mathcal{C}}^{s^{\prime}, *}(\mathbb{Z} / 2, \mathbb{Z} / 2)$ then the following relations hold:

$$
\mathbb{S} q^{s} \alpha=\left\{\begin{array}{lll}
\alpha^{2} & \text { if } & s=s^{\prime} \\
0 & \text { if } & s>s^{\prime}
\end{array}\right.
$$

Further, May shows in [12] that the operations (2-2) satisfy the Cartan formula (1-7) and the Adem relations (1-4), without the relation $\mathbb{S} q^{0}=1$. Thus, the work of Liulevicius and May can be summarized by saying that, under the operations (2-2), the cohomology of an arbitrary cocommutative Hopf algebra over $\mathbb{Z} / 2$ becomes an $\mathcal{H}$-algebra, in the sense of Definition 1.1.

The operation $\mathbb{S} q^{0}$ in (2-2) has an interesting description. Since the Hopf algebra $\mathcal{C}$ is cocommutative one can define the degree-halving "Verschiebung" homomorphism $V: \mathcal{C} \rightarrow \mathcal{C}$. (If $\mathcal{C}$ is locally finite one can describe $V$ as dual to the Frobenius mapping 
on the dual of $\mathcal{C}$.) The Verschiebung satisfies: $V(x y)=V(x) V(y)$ for all $x, y \in \mathcal{C}$, and so induces a homomorphism of cohomology groups:

$$
\operatorname{Ext}(V): \operatorname{Ext}_{\mathcal{C}}^{s, t}(\mathbb{Z} / 2, \mathbb{Z} / 2) \rightarrow \operatorname{Ext}_{\mathcal{C}}^{s, 2 t}(\mathbb{Z} / 2, \mathbb{Z} / 2), \quad s \geq 0, t \geq 0 .
$$

It is not hard to show that the operation $\mathbb{S} q^{0}$ of (2-2) is given by:

$$
\mathbb{S} q^{0}=\operatorname{Ext}(V) .
$$

A proof can be found in [22, Proposition 1.114].

In the paper [1], JF Adams invented his celebrated spectral sequence that converges to the stable homotopy groups of spheres, completed at $p=2$. Adams showed that the $E_{2}$-term of his spectral sequence could be identified with $\operatorname{Ext}_{\mathcal{A}}(\mathbb{Z} / 2, \mathbb{Z} / 2)$, the cohomology ring of the classical, singly-graded Steenrod algebra $\mathcal{A}$. This cohomology ring is still largely unknown. In the paper [19] the present writer studied the relationship of the cohomology of the Steenrod algebra $\mathcal{A}$ to the action of $\mathcal{A}$ on the polynomial rings $P^{\otimes s}$. The present work originated in some of the ideas of that paper. We review here the parts that are relevant.

If $\mathcal{C}$ is any algebra (assumed graded here), and if $M, N$, and $R$ are (graded) left $\mathcal{C}-$ modules, Yoneda defines in [27] the "composition pairing". This is a degree-preserving map of bigraded vector spaces:

$$
\operatorname{Ext}_{\mathcal{C}}(N, R) \otimes \operatorname{Ext}_{\mathcal{C}}(M, N) \rightarrow \operatorname{Ext}_{\mathcal{C}}(M, R) .
$$

Of interest to us now are the special cases:

$$
\operatorname{Ext}_{\mathcal{A}}\left(P^{\otimes s}, \mathbb{Z} / 2\right) \otimes \operatorname{Ext}_{\mathcal{A}}\left(\mathbb{Z} / 2, P^{\otimes s}\right) \rightarrow \operatorname{Ext}_{\mathcal{A}}(\mathbb{Z} / 2, \mathbb{Z} / 2)
$$

for all $s \geq 0$. In [19] this writer defined for each $s \geq 0$ a particular cohomology class:

$$
\sigma_{s} \in \mathrm{Ext}_{\mathcal{A}}^{s, s}\left(\mathbb{Z} / 2, P^{\otimes s}\right),
$$

and defined a homomorphism of graded $\mathbb{Z} / 2$-vector spaces, of degree $s$ :

$$
f_{s}: \operatorname{Ext}_{\mathcal{A}}^{0, *}\left(P^{\otimes s}, \mathbb{Z} / 2\right) \rightarrow \operatorname{Ext}_{\mathcal{A}}^{s, *}(\mathbb{Z} / 2, \mathbb{Z} / 2)
$$

by setting:

$$
f_{s}(\alpha)=\alpha \circ \sigma_{s}
$$

for each $\alpha \in \operatorname{Ext}^{0, *}\left(P^{\otimes s}, \mathbb{Z} / 2\right)$. Here we are writing $\alpha \circ \sigma_{s}$ for the image of $\alpha \otimes \sigma_{s}$ under the composition pairing (2-7). We can make the identifications:

$$
\operatorname{Ext}_{\mathcal{A}}^{0, *}\left(P^{\otimes s}, \mathbb{Z} / 2\right)=\operatorname{Hom}_{\mathcal{A}}^{*}\left(P^{\otimes s}, \mathbb{Z} / 2\right)=\left(\Gamma_{s, *}\right)^{\mathcal{A}},
$$


where $\left(\Gamma_{s, *}\right)^{\mathcal{A}}$ is an in (1-12). Using (2-11) and our definition (1-9) of the bigrading on $\Gamma$, we see that for each $s \geq 0$ the mapping (2-9) can be interpreted as a homomorphism of graded vector spaces of degree 0 :

$$
f_{s}:\left(\Gamma_{s, *}\right)^{\mathcal{A}} \rightarrow \operatorname{Ext}_{\mathcal{A}}^{s, *}(\mathbb{Z} / 2, \mathbb{Z} / 2) .
$$

In particular it is easy to compute $f_{1}$, and we have done so in [19]. This mapping is an isomorphism of vector spaces, and is given by:

$$
f_{1}\left(\gamma_{2^{i}-1}\right)=h_{i}, \quad i \geq 0 .
$$

Here $h_{i} \in \operatorname{Ext}_{\mathcal{A}}^{1,2^{i}}(\mathbb{Z} / 2, \mathbb{Z} / 2)$ is the well-known generator: the element represented by $\xi_{1}^{2^{i}}$ in the cobar construction on the dual of $\mathcal{A}$.

The collection $\left\{f_{s} \mid s \geq 0\right\}$ can be interpreted as a homomorphism of bigraded vector spaces:

$$
f: \Gamma^{\mathcal{A}} \rightarrow \operatorname{Ext}_{\mathcal{A}}(\mathbb{Z} / 2, \mathbb{Z} / 2) .
$$

of degree $(0,0)$. In [19] we show that $f$ is actually a homomorphism of bigraded algebras [19, Proposition 4.2].

Knowing that $\operatorname{Ext}_{\mathcal{A}}(\mathbb{Z} / 2, \mathbb{Z} / 2)$ is an $\mathcal{H}$-algebra, and knowing that $f$ is a homomorphism of algebras, one is naturally led to ask the following question.

Question 2.1 Is it possible to define an action of $\mathcal{H}$ on $\Gamma^{\mathcal{A}}$ in such a way that $\Gamma^{\mathcal{A}}$ is an $\mathcal{H}$-algebra, and $f$ becomes a morphism of $\mathcal{H}$-algebras?

Since we do not know a vector basis for $\Gamma^{\mathcal{A}}$, Question 2.1 is probably unanswerable, unless one asks first an apparently easier question.

Question 2.2 Is it possible to define an action of $\mathcal{H}$ on $\Gamma$, in such a way that:

(1) $\Gamma$ becomes an $\mathcal{H}$-algebra;

(2) the subalgebra $\Gamma^{\mathcal{A}} \subseteq \Gamma$ is stable under the action of $\mathcal{H}$, so that $\Gamma^{\mathcal{A}}$ also becomes an $\mathcal{H}$-algebra;

(3) $f$ is a morphism of $\mathcal{H}$-algebras.

We will see that the answer to both questions is almost certainly no.

Observe that one has in $\operatorname{Ext}_{\mathcal{A}}(\mathbb{Z} / 2, \mathbb{Z} / 2)$ the relations $\mathbb{S q}^{0}\left(h_{i}\right)=h_{i+1}$ for all $i \geq 0$. So in view of (2-13), the requirement that $f$ be a $\mathcal{H}$-linear map forces the equation 
$\mathbb{S} q^{0}\left(\gamma_{2^{i}-1}\right)=\gamma_{2^{i+1}-1}$ for each $i \geq 0$. Trying to answer Question 2.2 in the affirmative one is therefore led to a provisional definition:

$$
\mathbb{S} q^{0}\left(\gamma_{k}\right)=\gamma_{2 k+1}, \quad k \geq 0 .
$$

On the other hand the requirement that $\Gamma$ be an $\mathcal{H}$-algebra forces:

$$
\mathbb{S} q^{l}\left(\gamma_{k}\right)=\left\{\begin{array}{cl}
\gamma_{k} \times \gamma_{k}, & \text { if } l=1 \\
0 & \text { if } l>1
\end{array}\right.
$$

Now, the equation $\mathbb{S} q^{1} \mathbb{S} q^{1}=0$ is one of the Adem relations that define the algebra $\mathcal{H}$. Calculating from the above formulas and imposing the Cartan formula we get:

$$
\begin{aligned}
\mathbb{S} q^{1}\left(\mathbb{S} q^{1} \gamma_{k}\right) & =\mathbb{S} q^{1} \gamma_{k} \times \mathbb{S} q^{0} \gamma_{k}+\mathbb{S} q^{0} \gamma_{k} \times \mathbb{S} q^{1} \gamma_{k} \\
& =\gamma_{k} \times \gamma_{k} \times \gamma_{2 k+1}+\gamma_{2 k+1} \times \gamma_{k} \times \gamma_{k} .
\end{aligned}
$$

The result of this calculation is surely not zero in $\Gamma$, so the attempt via (2-15) and (2-16) to define an action of $\mathcal{H}$ has failed.

The attempt fails for yet another reason. The squaring operations on $\Gamma$ that are defined by (2-15) and (2-16), and by the Cartan formula, fail to carry $\Gamma^{\mathcal{A}}$ to $\Gamma^{\mathcal{A}}$. For example, one checks easily that $\gamma_{1} \times \gamma_{2}+\gamma_{2} \times \gamma_{1}$ lies in $\Gamma^{\mathcal{A}}$. Then (2-15) and (2-16) and the Cartan formula give:

(2-18) $\mathbb{S} q^{1}\left(\gamma_{1} \times \gamma_{2}+\gamma_{2} \times \gamma_{1}\right)=\gamma_{1} \times \gamma_{1} \times \gamma_{5}+\gamma_{3} \times \gamma_{2} \times \gamma_{2}+\gamma_{2} \times \gamma_{2} \times \gamma_{3}+\gamma_{5} \times \gamma_{1} \times \gamma_{1}$.

But the element on the right-hand side of (2-18) does not lie in $\Gamma^{\mathcal{A}}$ : it detects the polynomial $S q^{1}\left(t_{1}^{3} t_{2}^{2} t_{3}\right) \in P^{\otimes 3}$. So the obvious attempt to answer Question 2.2 in the affirmative does not succeed.

How could our goals be modified to give a positive result? A moment's consideration shows that if (2-17) were interpreted, not as an equation in $\Gamma$, but rather as an equation in $\mathbb{Z} / 2 \otimes_{\Sigma} \Gamma$, then the right hand side would read "zero", as required by the Adem relations in $\mathcal{H}$. Similarly, if (2-18) were interpreted as an equation in $\mathbb{Z} / 2 \otimes_{\Sigma} \Gamma$ then it would simply read $S q^{1}(0)=0$, and would not provide any obstruction to our defining an action of $\mathcal{H}$ on $\left(\mathbb{Z} / 2 \otimes_{\Sigma} \Gamma\right)^{\mathcal{A}}$. So we are led to modify Questions 2.1 and 2.2.

Question 2.3 Is it possible to define an action of $\mathcal{H}$ on $\mathbb{Z} / 2 \otimes_{\Sigma} \Gamma$, in such a way that:

(1) Equation (2-15) is satisfied;

(2) $\mathbb{Z} / 2 \otimes_{\Sigma} \Gamma$ becomes an $\mathcal{H}$-algebra;

(3) the subalgebra $\left(\mathbb{Z} / 2 \otimes_{\Sigma} \Gamma\right)^{\mathcal{A}}$ is stable under the action of $\mathcal{H}$, so that $\left(\mathbb{Z} / 2 \otimes_{\Sigma} \Gamma\right)^{\mathcal{A}}$ becomes an $\mathcal{H}$-algebra. 
In the remainder of the paper we will prove Theorem 1.3 and Theorem 1.4 and so answer this question in the affirmative.

\section{Structure of the bigraded Steenrod algebra}

We would like to construct proofs of Theorem 1.3 and Theorem 1.4 that are free of computation. For this purpose we develop some information in this section about the structure of the bigraded Steenrod algebra $\mathcal{H}$. In fact, knowing that the operations (2-2) satisfy the Cartan formula (1-7), one is naturally led to ask if the association $\mathrm{S} q^{k} \rightarrow \sum_{i+j=k} \mathbb{S} q^{i} \otimes \mathbb{S} q^{j}$ extends to some kind of coproduct on $\mathcal{H}$ that is compatible with the multiplication. The precise structure one needs to accommodate such a coproduct has been worked out in [21], and the first few definitions and results in this section are quoted from that paper. The main result of this section is Equation (3-5).

Definition 3.1 Fix a commutative ground ring $k$. By a " $k$-algebra with coproducts" we mean a bigraded $k$-algebra $\mathcal{D}=\left\{\mathcal{D}_{s, t} \mid s, t \geq 0\right\}$, together with degree-preserving $k$-linear mappings $\epsilon: \mathcal{D}_{*, t} \rightarrow k$ and $\psi_{t}: \mathcal{D}_{*, t} \rightarrow \mathcal{D}_{*, t} \otimes \mathcal{D}_{*, t}$, defined for each $t \geq 0$. We impose the following requirements.

(1) For each $t \geq 0, \mathcal{D}_{*, t}$ is a graded coalgebra, with counit $\epsilon_{t}$ and coproduct $\psi_{t}$.

(2) The algebra unit $\eta: k \rightarrow \mathcal{D}_{*, 0}$ is a map of coalgebras.

(3) For each pair of integers $t, t^{\prime} \geq 0$, the multiplication $\mu: \mathcal{D}_{*, t} \otimes \mathcal{D}_{*, t^{\prime}} \rightarrow \mathcal{D}_{*, t+t^{\prime}}$ is a map of graded coalgebras.

Let $\mathcal{H}$ be the bigraded Steenrod algebra, as defined in Section 1. The main result of $[21]$ is the following proposition.

Proposition 3.2 The bigraded Steenrod algebra $\mathcal{H}$ admits a unique structure as a $\mathbb{Z} / 2$-algebra with coproducts, with $\psi_{1}: \mathcal{H}_{*, 1} \rightarrow \mathcal{H}_{*, 1} \otimes \mathcal{H}_{*, 1}$ given by:

$$
\psi_{1}\left(\mathbb{S} q^{l}\right)=\sum_{i+j=l} \mathbb{S} q^{i} \otimes \mathbb{S} q^{j}
$$

Note that the counit $\epsilon_{t}: \mathcal{H}_{*, t} \rightarrow \mathbb{Z} / 2$ is necessarily given by:

$$
\epsilon\left(\mathbb{S} q^{l_{1}} \mathbb{S} q^{l_{2}} \ldots S \mathbb{S} q^{l_{t}}\right)= \begin{cases}1 & \text { if } l_{1}=l_{2}=\ldots l_{t}=0, \\ 0 & \text { otherwise. }\end{cases}
$$

In Section 1 we defined $\mathcal{H}$ by giving a presentation for it as an algebra. We will find it useful to "present" $\mathcal{H}$ as an algebra with coproducts. To this end, we write $\mathcal{F}$ for the 
free associative algebra ("tensor algebra") generated by the symbols $\left\{\mathbb{S} q^{l} \mid l \geq 0\right\}$, and bigraded by the rule $\operatorname{deg}\left(\mathbb{S} q^{l}\right)=(l, 1)$. For each $t \geq 0$ we make $\mathcal{F}_{*, t}$ into a coalgebra, defining the coproduct $\psi_{t}: \mathcal{F}_{*, t} \rightarrow \mathcal{F}_{*, t} \otimes \mathcal{F}_{*, t}$ by:

$$
\psi_{t}\left(\mathbb{S} q^{l_{1}} \ldots \mathbb{S} q^{l_{t}}\right)=\sum_{i_{1}+j_{1}=l_{1}} \ldots \sum_{i_{t}+j_{t}=l_{t}}\left(\mathbb{S} q^{i_{1}} \ldots \mathbb{S} q^{i_{t}}\right) \otimes\left(\mathbb{S} q^{j_{1}} \ldots \mathbb{S} q^{j_{t}}\right)
$$

We define the counit $\epsilon: \mathcal{F}_{*, t} \rightarrow \mathbb{Z} / 2$ by a formula that is formally identical to (3-2). It is easy to check that these definitions make $\mathcal{F}_{*, t}$ into a graded coalgebra, for each $t \geq 0$. It is also easy to see that the product $\mathcal{F}_{*, t} \otimes \mathcal{F}_{*, t^{\prime}} \rightarrow \mathcal{F}_{*, t+t^{\prime}}$ is a morphism of coalgebras, for all $t, t^{\prime} \geq 0$. We conclude that our definitions make $\mathcal{F}$ into an algebra with coproducts.

We write $\pi: \mathcal{F} \rightarrow \mathcal{H}$ for the unique homomorphism of bigraded algebras that carries $\mathbb{S} q^{l} \in \mathcal{F}$ to $\mathbb{S} q^{l} \in \mathcal{H}$. For each $t \geq 0$ it is clear that $\pi: \mathcal{F}_{*, t} \rightarrow \mathcal{H}_{*, t}$ is a morphism of coalgebras; hence, that $\pi: \mathcal{F} \rightarrow \mathcal{H}$ is a morphism of algebras with coproducts.

For each pair of integers $a, b$ with $0 \leq a<2 b$ we define an element $R(a, b)$ of $\mathcal{F}$ by writing:

$$
R(a, b)=\mathbb{S} q^{a} \mathbb{S} q^{b}+\sum_{j \geq 0}\left(\begin{array}{c}
b-1-j \\
a-2 j
\end{array}\right) \mathbb{S} q^{a+b-j} \mathbb{S} q^{j} \quad 0 \leq a<2 b .
$$

We write $\mathcal{I} \subset \mathcal{F}$ for the homogeneous, two-sided ideal that is generated by the elements (3-4). For each $t \geq 0$ we consider the following diagram of vector space homomorphisms:

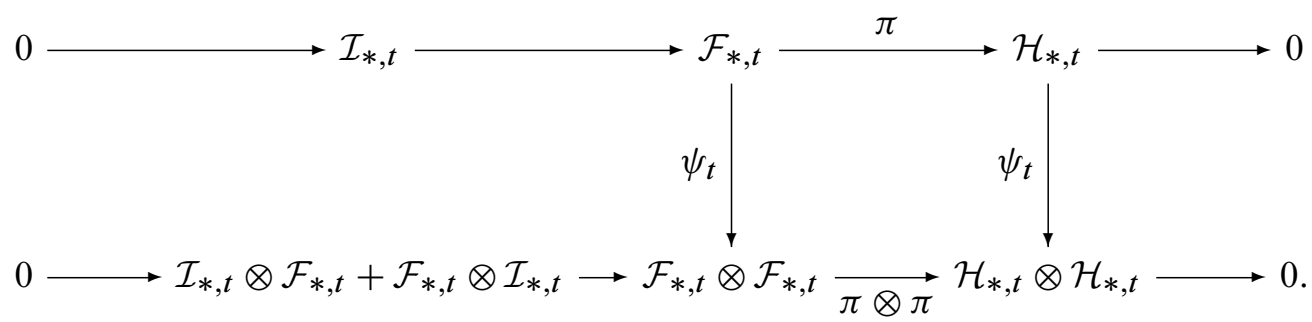

The exactness of the top row is the definition of $\mathcal{H}$; the exactness of the bottom row follows by elementary linear algebra, and the commutativity of the square follows from (3-3) and Proposition 3.2. It follows that for each $t \geq 0$ we have:

$$
\psi_{t}\left(\mathcal{I}_{*, t}\right) \subset \mathcal{I}_{*, t} \otimes \mathcal{F}_{*, t}+\mathcal{F}_{*, t} \otimes \mathcal{I}_{*, t}
$$

This result will be useful to us in the next section, in defining the action of $\mathcal{H}$ on $\left(\mathbb{Z} / 2 \otimes_{\Sigma} \Gamma\right)$. 


\section{Action of $\mathcal{H}$ on $\mathbb{Z} / 2 \otimes_{\Sigma} \Gamma$}

The purpose of this section is to prove Theorem 1.3.

We begin by establishing vector bases for $\Gamma$ and $\mathbb{Z} / 2 \otimes_{\Sigma} \Gamma$. As is well known one has $H_{*}(B(\mathbb{Z} / 2), \mathbb{Z} / 2)=\operatorname{Span}\left\{\gamma_{k} \mid k \geq 0\right\}$, with $\gamma_{k} \in H_{k}(B(\mathbb{Z} / 2), \mathbb{Z} / 2)$. This is an algebra of divided powers under Pontryagin product. Thus our choice of the letter "gamma" to denote the generators. However, this Pontryagin product will play no further role in our work. On the other hand the cross product (1-10) will be crucial for us. From the Eilenberg-Zilber and Künneth theorems we have:

$$
H_{*}\left(B(\mathbb{Z} / 2)^{\times s}, \mathbb{Z} / 2\right)=\operatorname{Span}\left\{\gamma_{i_{1}} \times \gamma_{i_{2}} \times \cdots \times \gamma_{i_{s}} \mid i_{1}, i_{2}, \ldots i_{s} \geq 0\right\} .
$$

It follows from our definition (1-9) that $\Gamma=\operatorname{Tens}\left\{\gamma_{0}, \gamma_{1}, \ldots, \gamma_{s}, \ldots\right\}$, the free associative algebra on the symbols $\left\{\gamma_{k} \mid k \geq 0\right\}$, bigraded according to the rule: $\operatorname{deg}\left(\gamma_{k}\right)=$ $(1, k+1)$.

The effect of dividing $\Gamma_{s, *}$ by the action of the symmetric group $\Sigma_{s}$ is to identify any two elements of the basis (4-1) that can be obtained from one another by permutation of the factors. So we have from the definition (1-13):

$$
\mathbb{Z} / 2 \otimes_{\Sigma} \Gamma=\mathbb{Z} / 2\left[\gamma_{0}, \gamma_{1}, \ldots, \gamma_{k}, \ldots\right]
$$

the polynomial algebra on the symbols $\left\{\gamma_{k} \mid k \geq 0\right\}$, with $\operatorname{deg}\left(\gamma_{k}\right)=(1, k+1)$.

Our next task is to define an action of $\mathcal{H}$ on $\mathbb{Z} / 2 \otimes_{\Sigma} \Gamma$ that satisfies the conditions of Theorem 1.3. We recall from Section 3 the free algebra $\mathcal{F}$ on the symbols $\left\{\mathbb{S} q^{l} \mid l \geq 0\right\}$. We will begin by defining an appropriate action of $\mathcal{F}$ on $\mathbb{Z} / 2 \otimes_{\Sigma} \Gamma$, and then show that this action passes to an action of the quotient algebra $\mathcal{H}=\mathcal{F} / \mathcal{I}$.

For the action of $\mathcal{H}$ on $\mathbb{Z} / 2 \otimes_{\Sigma} \Gamma$ we will need:

$$
\mathbb{S} q^{l} \gamma_{k}= \begin{cases}\gamma_{2 k+1} & \text { if } l=0 \\ \gamma_{k}^{2} & \text { if } l=1 \\ 0 & \text { if } l>1\end{cases}
$$

So we impose these conditions on the action of $\mathcal{F}$. We will also want the action of $\mathcal{H}$ to obey the Cartan formula (1-7), so we begin by imposing this requirement on the action of $\mathcal{F}$. We are forced to set:

$$
\mathbb{S} q^{l}\left(\gamma_{k}^{a}\right)=\left(\begin{array}{l}
a \\
l
\end{array}\right) \gamma_{k}^{2 l} \gamma_{2 k+1}^{a-l} \quad \forall a, k, l \geq 0 .
$$


Imposing the Cartan formula one more time leads to our definition of the action of $\mathcal{F}$ on $\mathbb{Z} / 2 \otimes_{\Sigma} \Gamma$ :

$$
\begin{aligned}
& \mathbb{S} q^{l}\left(\gamma_{0}^{a_{0}} \gamma_{1}^{a_{1}} \ldots \gamma_{m}^{a_{m}}\right) \\
& \quad=\sum_{i_{0}+\ldots i_{m}=l}\left(\begin{array}{c}
a_{0} \\
i_{0}
\end{array}\right)\left(\begin{array}{c}
a_{1} \\
i_{1}
\end{array}\right) \ldots\left(\begin{array}{c}
a_{m} \\
i_{m}
\end{array}\right)\left(\gamma_{0}^{i_{0}} \ldots \gamma_{m}^{i_{m}}\right)^{2}\left(\gamma_{1}^{a_{0}-i_{0}} \gamma_{3}^{a_{1}-i_{1}} \ldots \gamma_{2 m+1}^{a_{m}-i_{m}}\right)
\end{aligned}
$$

for all $l, m \geq 0, a_{0}, \ldots a_{m} \geq 0$.

We arrived at (4-5) by applying the Cartan formula to particular kinds of products. However, one must check that the Cartan formula holds in general.

Proposition 4.1 The action of $\mathcal{F}$ on $\mathbb{Z} / 2 \otimes_{\Sigma} \Gamma$ that is defined by (4-5) obeys the Cartan formula (1-7) for all $l \geq 0$ and all $\alpha, \beta \in \mathbb{Z} / 2 \otimes_{\Sigma} \Gamma$.

The proof is a simple computation that uses a well-known property of binomial coefficients. More generally we have the following result.

Proposition 4.2 Suppose the coproduct $\psi_{t}: \mathcal{F}_{*, t} \rightarrow \mathcal{F}_{*, t} \otimes \mathcal{F}_{*, t}$ maps a given $X \in \mathcal{F}_{*, t}$ according to:

$$
\psi_{t}(X)=\sum_{i} X_{i} \otimes X_{i}^{\prime}
$$

Then for any $\alpha, \beta \in \mathbb{Z} / 2 \otimes_{\Sigma} \Gamma$ one has:

$$
X(\alpha \beta)=\sum_{i} X_{i}(\alpha) X_{i}^{\prime}(\beta)
$$

The proof is by induction on $t$. The case $t=1$ is Proposition 4.1. One carries out the inductive step by appeal to the fact that the multiplication $\mathcal{F}_{*, 1} \otimes \mathcal{F}_{*, t-1} \rightarrow \mathcal{F}_{*, t}$ is a morphism of coalgebras.

Corollary 4.3 The action of $\mathcal{F}$ on $\mathbb{Z} / 2 \otimes_{\Sigma} \Gamma$ satisfies:

$$
\mathbb{S} q^{l}\left(\alpha^{2^{s}}\right)=\left\{\begin{array}{l}
\left(\mathbb{S} q^{l / 2^{s}} \alpha\right)^{2^{s}} \text { if } 2^{s} \mid l, \\
0 \text { otherwise, }
\end{array}\right.
$$

for all $l, s \geq 0$ and all $\alpha \in \mathbb{Z} / 2 \otimes_{\Sigma} \Gamma$. 
This follows immediately from Proposition 4.1 and the commutativity of the algebra $\mathbb{Z} / 2 \otimes_{\Sigma} \Gamma$. As an immediate consequence of this result, and Equation (4-3) we have for all $l, k, s \geq 0$ :

$$
\mathbb{S} q^{l}\left(\gamma_{k}^{2^{s}}\right)= \begin{cases}\gamma_{2 k+1}^{2^{s}} & \text { if } l=0 \\ \gamma_{k}^{2^{s+1}} & \text { if } l=2^{s} \\ 0 & \text { otherwise }\end{cases}
$$

Corollary 4.4 Let $Y \in \mathcal{F}$ be any monomial in the generators $\left\{\mathbb{S} q^{l} \mid l \geq 0\right\}$, and let $j \geq 0$ be an integer. Then either $Y\left(\gamma_{j}\right)=0$ or there exist integers $k \geq j$ and $s \geq 0$ for which $Y\left(\gamma_{j}\right)=\gamma_{k}^{2^{s}}$.

This follows from (4-9) by induction on the length of the monomial $Y$ in the generators.

We want to pass from the action of $\mathcal{F}$ on $\mathbb{Z} / 2 \otimes_{\Sigma} \Gamma$ that we have just defined, to an action of $\mathcal{H}$. So we need to show that:

$$
\mathcal{I}\left(\mathbb{Z} / 2 \otimes_{\Sigma} \Gamma\right)=0,
$$

where $\mathcal{I}$ is the two-sided ideal of $\mathcal{F}$ that is generated by the elements (3-4). We start with the following proposition.

Proposition 4.5 Let $a, b, s, k$ be integers with $0 \leq a<2 b$, and $0 \leq k, s$. Then:

$$
R(a, b) \gamma_{k}^{2^{s}}=0 .
$$

Proof Consider first the case $b \neq 2^{s}$. Then we must show that:

$$
\sum_{j \geq 0}\left(\begin{array}{c}
b-1-j \\
a-2 j
\end{array}\right) \mathbb{S} q^{a+b-j} \mathbb{S} q^{j}\left(\gamma_{k}^{2^{s}}\right)=0 .
$$

But according to (4-9) the only terms in the sum that could possibly be nonzero are those for which either $j=0$ or $j=2^{s}$. We will show that both terms are in fact zero. For the case $j=0$ we must show that:

$$
\left(\begin{array}{c}
b-1 \\
a
\end{array}\right) \mathbb{S} q^{a+b}\left(\gamma_{2 k+1}^{2^{s}}\right)=0
$$

We note that $\mathbb{S} q^{a+b}\left(\gamma_{2 k+1}^{2^{s}}\right)$ could be nonzero only if $a+b=2^{s}$. But if $a+b=2^{s}$ and $b \neq 2^{s}$ then it is easy to show by using properties of binomial coefficients modulo 2, as in [23, Lemma 2.6], that $\left(\begin{array}{c}b-1 \\ a\end{array}\right)=0$. So (4-13) is true. We must next consider the term $j=2^{s}$ in the sum (4-12). We must show that:

$$
\left(\begin{array}{c}
b-1-2^{s} \\
a-2^{s+1}
\end{array}\right) \mathbb{S} q^{a+b-2^{s}}\left(\gamma_{k}^{2^{s+1}}\right)=0 .
$$


According to (4-9) we need only consider the cases $a+b=2^{s}$ and $a+b=2^{s}+2^{s+1}$. In the former case (4-14) is surely true, because the lower entry in the binomial coefficient is negative. In the latter case the lower entry in the binomial coefficient is again negative, because the statements $a+b=2^{s}+2^{s+1}$ and $a<2 b$ imply $a<2^{s+1}$. So (4-11) has been established in the case $b \neq 2^{s}$. We consider finally the case $b=2^{s}$. We have from (4-9):

$$
\mathbb{S} q^{a} \mathbb{S} q^{2^{s}}\left(\gamma_{k}^{2^{s}}\right)=\mathbb{S} q^{a}\left(\gamma_{k}^{2^{s+1}}\right)= \begin{cases}\gamma_{2 k+1}^{2^{s+1}} & \text { if } a=0 \\ 0 & \text { otherwise }\end{cases}
$$

(The second case of (4-9) will not occur here, since we are assuming $a<2 b$.) So in order to show that (4-11) holds we must show:

$$
\sum_{j \geq 0}\left(\begin{array}{c}
2^{s}-1-j \\
a-2 j
\end{array}\right) \mathbb{S} q^{a+2^{s}-j} \mathbb{S} q^{j}\left(\gamma_{k}^{2^{s}}\right)= \begin{cases}\gamma_{2 k+1}^{2^{s+1}} & \text { if } a=0, \\
0 & \text { if } 0<a<2^{s+1}\end{cases}
$$

The case $a=0$ is obvious, since only the term $j=0$ will contribute to the sum. For the case $a>0$ we know from (4-9) that $\mathbb{S} q^{j}\left(\gamma_{k}^{2^{s}}\right)$ can be nonzero only if either $j=0$ or $j=2^{s}$. But if $j=2^{s}$ then $a-2 j<0$ and the binomial coefficient vanishes. So we need only consider the term in the sum for which $j=0$. It will suffice to show that $\mathbb{S} q^{a+2^{s}} \mathbb{S} q^{0}\left(\gamma_{k}^{2^{s}}\right)=\mathbb{S} q^{a+2^{s}}\left(\gamma_{2 k+1}^{2^{s}}\right)=0$. But since $a>0$ this is obvious from (4-9).

We continue toward a proof of (4-10) with the following proposition.

Proposition 4.6 Let $X \in \mathcal{I}$ be any element of the ideal $\mathcal{I}$, and let $j \geq 0$. Then $X\left(\gamma_{j}\right)=0$.

Proof It suffices to consider the case in which $X=R(a, b) Y$ for any $Y \in \mathcal{F}$ and $0 \leq a<2 b$. If $Y\left(\gamma_{j}\right)=0$ we are done. Otherwise we have from Corollary 4.4 that $X\left(\gamma_{j}\right)=R(a, b)\left(\gamma_{k}^{2^{s}}\right)$ for some integers $k, s \geq 0$, so our result follows from Proposition 4.5 .

Now we can prove (4-10).

Proposition 4.7 Let $\alpha$ be any element of $\mathbb{Z} / 2 \otimes_{\Sigma} \Gamma$, and suppose that $X \in \mathcal{F}$ belongs to the ideal $\mathcal{I}$. Then $X(\alpha)=0$.

Proof Supposing $\alpha \in\left(\mathbb{Z} / 2 \otimes_{\Sigma} \Gamma\right)_{s, *}$, we will prove the result by induction on $s$. The case $s=1$ is just the previous proposition. Suppose the result proved for all $\alpha \in\left(\mathbb{Z} / 2 \otimes_{\Sigma} \Gamma\right)_{r, *}$ for all $r$ with $1 \leq r<s$, for some $s \geq 2$. Given $\alpha \in\left(\mathbb{Z} / 2 \otimes_{\Sigma} \Gamma\right)_{s, *}$ 
we can write $\alpha=\gamma_{j} \beta$ for some $j \geq 0$ and some $\beta \in\left(\mathbb{Z} / 2 \otimes_{\Sigma} \Gamma\right)_{s-1, *}$. Further, by Equation (3-5) we can write: $\psi(X)=\sum_{i} X_{i} \otimes X_{i}^{\prime}$, where for each index $i$ either $X_{i} \in \mathcal{I}$ or $X_{i}^{\prime} \in \mathcal{I}$. If we then use the right-hand side of (4-7) to expand $X(\alpha)=X\left(\gamma_{j} \beta\right)$ it is clear from our inductive assumption that $X(\alpha)=0$.

Proof of Theorem 1.3 By Proposition 4.7, the action of $\mathcal{F}$ upon $\mathbb{Z} / 2 \otimes_{\Sigma} \Gamma$ that is defined by (4-5) passes to an action of the quotient algebra $\mathcal{H}=\mathcal{F} / \mathcal{I}$. This quotient action satisfies all the requirements of Theorem 1.3. For example, the action of $\mathcal{H}$ satisfies the Cartan formula, because the action of $\mathcal{F}$ does (Proposition 4.1). That we have satisfied the requirement that $\mathbb{S} q^{s} \alpha=\alpha^{2}$ if $\alpha \in\left(\mathbb{Z} / 2 \otimes_{\Sigma} \Gamma\right)_{s, *}$ is clear from (4-5). In fact, suppose $\alpha=\gamma_{0}^{a_{0}} \gamma_{1}^{a_{1}} \ldots \gamma_{m}^{a_{m}}$ with $a_{0}+\cdots+a_{m}=s$. Taking $l=s$ in (4-5) one sees that the product $\left(\begin{array}{l}a_{0} \\ i_{0}\end{array}\right)\left(\begin{array}{l}a_{1} \\ i_{1}\end{array}\right) \ldots\left(\begin{array}{l}a_{m} \\ i_{m}\end{array}\right)$ is nonzero if and only if $i_{0}=a_{0}, \ldots i_{m}=a_{m}$. So $\mathbb{S} q^{s} \alpha=\alpha^{2}$. The uniqueness of the action of $\mathcal{H}$ satisfying the requirements of Theorem 1.3 is also clear. In fact, since the action satisfies the Cartan formula (1-7), it is uniquely determined by the values of the $\mathbb{S} q^{l}$ upon the algebra generators $\gamma_{k}$, as specified in (4-3).

\section{Action of $\mathcal{H}$ on $\left(\mathbb{Z} / 2 \otimes_{\Sigma} \Gamma\right)^{\mathcal{A}}$}

The purpose of this section is to prove Theorem 1.4.

Recall from Section 1 that we have defined a right action of $\mathcal{A}$, the singly graded Steenrod algebra, on the bigraded vector space $\mathbb{Z} / 2 \otimes_{\Sigma} \Gamma$. We review here some details about this action. For each $s \geq 0, \mathcal{A}$ is acting on the right of $\left(\mathbb{Z} / 2 \otimes_{\Sigma} \Gamma\right)_{s, *}$, and lowers the internal degree. Since the action of $\mathcal{A}$ on $\mathbb{Z} / 2 \otimes_{\Sigma} \Gamma$ satisfies the Cartan formula (1-14), and since $\mathbb{Z} / 2 \otimes_{\Sigma} \Gamma$ is generated as an algebra by $\left(\mathbb{Z} / 2 \otimes_{\Sigma} \Gamma\right)_{1, *}$, the action of $\mathcal{A}$ on $\left(\mathbb{Z} / 2 \otimes_{\Sigma} \Gamma\right)_{s, *}$ for each $s \geq 0$ is completely determined by its action on $\left(\mathbb{Z} / 2 \otimes_{\Sigma} \Gamma\right)_{1, *}$. But this is just the right action of $\mathcal{A}$ on the homology of real projective space, so we record:

$$
\left(\gamma_{k}\right) S q^{p}=\left(\begin{array}{c}
k-p \\
p
\end{array}\right) \gamma_{k-p}, \quad k, p \geq 0 .
$$

Theorem 1.4 will follow immediately from the following technical result, which describes how the actions of $\mathcal{A}$ and $\mathcal{H}$ on $\mathbb{Z} / 2 \otimes_{\Sigma} \Gamma$ commute. The reader will notice the several places in the proof where we must use the commutativity of the algebra $\mathbb{Z} / 2 \otimes_{\Sigma} \Gamma$. The argument would not work if, rather than working in $\mathbb{Z} / 2 \otimes_{\Sigma} \Gamma$, we were working in $\Gamma$.

In the diagram accompanying Proposition 5.1 we intend the symbol $S q^{p / 2}$ to mean "zero" if $p$ is odd. 
Proposition 5.1 For all values of $s, t, l, p \geq 0$ the diagram commutes:

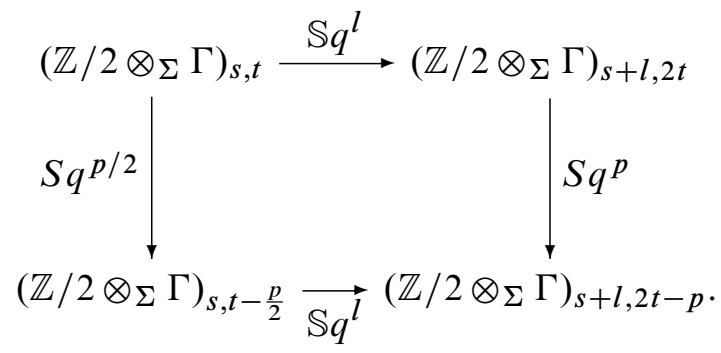

Proof We consider first the case in which $p$ is odd; we must show that for each $\alpha \in\left(\mathbb{Z} / 2 \otimes_{\Sigma} \Gamma\right)_{s, *}$ we have:

$$
\left(\mathbb{S} q^{l} \alpha\right) S q^{p}=0 \quad \text { if } l \geq 0, p \text { odd. }
$$

The proof is by induction on $s$. If $s=1$ we have $\alpha=\gamma_{k}$ for some $k \geq 0$. Since $\mathbb{S} q^{l}\left(\gamma_{k}\right)=0$ if $l>1$, we need to check only the cases $l=0,1$. If $l=0$ we have from $(5-1)$ :

$$
\left(\mathbb{S} q^{0} \gamma_{k}\right) S q^{p}=\left(\gamma_{2 k+1}\right) S q^{p}=\left(\begin{array}{c}
2 k+1-p \\
p
\end{array}\right) \gamma_{2 k+1-p} .
$$

The binomial coefficient is zero modulo 2 if $p$ is odd, so (5-3) is true. If $l=1$ we have $\left(\mathbb{S} q^{1} \gamma_{k}\right) S q^{p}=\left(\gamma_{k}^{2}\right) S q^{p}$. But if $p$ is odd the Cartan formula (1-14) implies that $\left(\gamma_{k}^{2}\right) S q^{p}=0$, so (5-3) is satisfied in this case as well. Now suppose we have proved (5-3) for all $\alpha \in\left(\mathbb{Z} / 2 \otimes_{\Sigma} \Gamma\right)_{r, *}$ for all $r$ with $1 \leq r<s$ for some $s \geq 2$. Let $\alpha \in\left(\mathbb{Z} / 2 \otimes_{\Sigma} \Gamma\right)_{s, *}$ be given. Then we can write $\alpha=\gamma_{k} \beta$ for some $k \geq 0$ and some $\beta \in\left(\mathbb{Z} / 2 \otimes_{\Sigma} \Gamma_{s-1, *}\right)$. Then $\mathbb{S} q^{l} \alpha=\left(\mathbb{S} q^{0} \gamma_{k}\right)\left(\mathbb{S} q^{l} \beta\right)+\left(\mathbb{S} q^{1} \gamma_{k}\right)\left(\mathbb{S} q^{l-1} \beta\right)$, so by $(1-14)$ :

$$
\begin{aligned}
\left(\mathbb{S} q^{l} \alpha\right) S q^{p} & =\sum_{a+b=p}\left[\left(\mathbb{S} q^{0} \gamma_{k}\right) S q^{a}\right] \cdot\left[\left(\mathbb{S} q^{l} \beta\right) S q^{b}\right] \\
& +\sum_{a+b=p}\left[\left(\mathbb{S} q^{1} \gamma_{k}\right) S q^{a}\right] \cdot\left[\left(\mathbb{S} q^{l-1} \beta\right) S q^{b}\right]
\end{aligned}
$$

But $p$ is odd, so that in each term of each sum, at least one of the integers $a, b$ must be odd. So by the inductive hypothesis we have $\left(\mathbb{S} q^{l} \alpha\right) S q^{p}=0$, as claimed.

We consider next the case in which $p$ is even; say $p=2 q$ for some $q \geq 0$. We will prove the commutativity of (5-2) by induction on $s$. If $s=1$ we need only consider the cases $l=0, l=1$. In case $l=0$ we find from (5-1):

$$
\left(\mathbb{S} q^{0} \gamma_{k}\right) S q^{p}=\left(\gamma_{2 k+1}\right) S q^{2 q}=\left(\begin{array}{c}
2 k+1-2 q \\
2 q
\end{array}\right) \gamma_{2 k+1-2 q} .
$$


On the other hand,

$$
\mathbb{S} q^{0}\left[\left(\gamma_{k}\right) S q^{\frac{p}{2}}\right]=\left(\begin{array}{c}
k-q \\
q
\end{array}\right) \mathbb{S} q^{0} \gamma_{k-q}=\left(\begin{array}{c}
k-q \\
q
\end{array}\right) \gamma_{2 k+1-2 q}
$$

The binomial coefficients on the right-hand sides of equations (5-5) and (5-6) are equal modulo 2, so we have shown commutativity of (5-2) if $s=1, l=0, p$ even. If $s=1, l=1$ and $p=2 q$ we use (1-8) and (1-14), and the commutativity of the algebra $\mathbb{Z} / 2 \otimes_{\Sigma} \Gamma: \quad\left(\mathbb{S} q^{1} \gamma_{k}\right) S q^{2 q}=\left(\gamma_{k}^{2}\right) S q^{2 q}=\left[\left(\gamma_{k}\right) S q^{q}\right]^{2}=\mathbb{S} q^{1}\left[\left(\gamma_{k}\right) S q^{q}\right]$. So the commutativity of (5-2) is established in this case as well. Now we proceed with the inductive step. Writing $p=2 q$ we assume that the equation

$$
\left(\mathbb{S} q^{l} \alpha\right) S q^{2 q}=\mathbb{S} q^{l}\left[(\alpha) S q^{q}\right]
$$

has been established for all $\alpha \in\left(\mathbb{Z} / 2 \otimes_{\Sigma} \Gamma\right)_{r, *}$, for all $r$ with $1 \leq r \leq s-1$, for some $s \geq 2$. Let $\alpha \in\left(\mathbb{Z} / 2 \otimes_{\Sigma} \Gamma\right)_{s, *}$ be given. We write $\alpha=\gamma_{k} \beta$ for some $k \geq 0$ and some $\beta \in\left(\mathbb{Z} / 2 \otimes_{\Sigma} \Gamma_{s-1, *}\right)$. We compute using the Cartan formulas (1-7) and (1-14):

$\left(\mathbb{S} q^{l} \alpha\right) S q^{2 q}=\left(\mathbb{S} q^{0} \gamma_{k} \cdot \mathbb{S} q^{l} \beta+\mathbb{S} q^{1} \gamma_{k} \cdot \mathbb{S} q^{l-1} \beta\right) S q^{2 q}$

$$
=\sum_{a+b=q}\left[\left(\mathbb{S} q^{0} \gamma_{k}\right) S q^{2 a} \cdot\left(\mathbb{S} q^{l} \beta\right) S q^{2 b}+\left(\mathbb{S} q^{1} \gamma_{k}\right) S q^{2 a} \cdot\left(\mathbb{S} q^{l-1} \beta\right) S q^{2 b}\right],
$$

where we have also used the newly established (5-3). So by (1-7) and (1-14) and the inductive hypothesis we have:

$$
\begin{aligned}
\left(\mathbb{S} q^{l} \alpha\right) S q^{2 q} & =\sum_{a+b=q}\left[S q^{0}\left(\gamma_{k} S q^{a}\right) \cdot \mathbb{S} q^{l}\left(\beta S q^{b}\right)+\mathbb{S} q^{1}\left(\gamma_{k} S q^{a}\right) \cdot \mathbb{S} q^{l-1}\left(\beta S q^{b}\right)\right] \\
& =\sum_{a+b=q} \mathbb{S} q^{l}\left(\gamma_{k} S q^{a} \cdot \beta S q^{b}\right)=\mathbb{S} q^{l} \sum_{a+b=q}\left(\gamma_{k} S q^{a} \cdot \beta S q^{b}\right) \\
& =\mathbb{S} q^{l}\left[\left(\gamma_{k} \beta\right) S q^{q}\right]=\mathbb{S} q^{l}\left[(\alpha) S q^{q}\right] .
\end{aligned}
$$

This completes the inductive proof of (5-7), and the proof of Proposition 5.1.

Proof of Theorem 1.4 That the subalgebra $\left(\mathbb{Z} / 2 \otimes_{\Sigma} \Gamma\right)^{\mathcal{A}} \subseteq \mathbb{Z} / 2 \otimes_{\Sigma} \Gamma$ is stable under the action of $\mathcal{H}$ follows immediately from Proposition 5.1.

\section{Sample computations}

We want to write down some interesting examples of the action of $\mathcal{H}$ on elements of $\left(\mathbb{Z} / 2 \otimes_{\Sigma} \Gamma\right)^{\mathcal{A}}$. First we need elements of $\left(\mathbb{Z} / 2 \otimes_{\Sigma} \Gamma\right)^{\mathcal{A}}$ ! One family is easily identified. A monomial $\alpha \in \mathbb{Z} / 2 \otimes_{\Sigma} \Gamma$ is called a "spike" if it has the form:

$$
\alpha=\gamma_{2^{p_{1}-1}} \gamma_{2^{p_{2}-1} \cdots \gamma_{2}{ }^{p_{s-1}}}
$$


for integers $p_{1}, p_{2} \cdots p_{s} \geq 0$. It is immediately clear from (5-1) that any spike is $\mathcal{A}-$ annihilated (here of course we are using properties of binomial coefficients modulo two, as in [23, Lemma 2.6]). Under the pairing of the vector space $\left(\left(\mathbb{Z} / 2 \otimes_{\Sigma} \Gamma\right)^{\mathcal{A}}\right)_{s, *}$ with its dual, the spikes detect the elements of $\mathbb{Z} / 2 \otimes_{\mathcal{A}}\left(P^{\otimes s}\right)^{\Sigma_{s}}$ that are called "symmetrized spikes" by Janfada and Wood in [8].

For any $l \geq 0$ we have from (4-3) and the Cartan formula:

$$
\mathbb{S} q^{l} \alpha=\sum_{0 \leq i_{1} \leq 1} \cdots \sum_{0 \leq i_{s} \leq 1} \mathbb{S} q^{i_{1}} \gamma_{2^{p_{1}-1}} \cdots \mathbb{S} q^{i_{s}} \gamma_{2^{p_{s}-1}}
$$

where the sum is over all sequences of zeros and ones $\left\{i_{1}, \cdots i_{s}\right\}$ with $i_{1}+\cdots i_{s}=l$. But $\mathbb{S} q^{0} \gamma_{2^{p}-1}=\gamma_{2^{p+1}-1}$ and $\mathbb{S} q^{1} \gamma_{2^{p}-1}=\left(\gamma_{2^{p}-1}\right)^{2}$, so it is clear from (6-2) that the operations of $\mathcal{H}$ upon $\left(\mathbb{Z} / 2 \otimes_{\Sigma} \Gamma\right)^{\mathcal{A}}$ carry spikes to linear combinations of spikes.

We have recorded this result in the interests of completeness; but the most interesting elements of $\left(\mathbb{Z} / 2 \otimes_{\Sigma} \Gamma\right)^{\mathcal{A}}$ are those which are not linear combinations of spikes. We get some examples by writing duals of some of the elements in $\mathbb{Z} / 2 \otimes_{\mathcal{A}}\left(P^{\otimes 3}\right)^{\Sigma_{3}}$ that are found in [8] by Janfada and Wood. Then we will see that the algebra $\mathcal{H}$ can act nontrivially on these dual classes. We will adopt from [8] the notation $\sigma(f)$ for the "symmetrization" of a monomial $f \in P^{\otimes s} . \sigma(f)$ is the smallest symmetric polynomial in $P^{\otimes s}$ that contains $f$ as a term.

Consider $\alpha \in\left(\mathbb{Z} / 2 \otimes_{\Sigma} \Gamma\right)_{3,10}$ defined by: $\alpha=\gamma_{3} \gamma_{2}^{2}+\gamma_{5} \gamma_{1}^{2}$. This element is clearly $\mathcal{A}$-annihilated, since we have $\left(\gamma_{3} \gamma_{2}^{2}\right) S q^{1}=\left(\gamma_{5} \gamma_{1}^{2}\right) S q^{1}=0$ and $\left(\gamma_{3} \gamma_{2}^{2}\right) S q^{2}=$ $\left(\gamma_{5} \gamma_{1}^{2}\right) S q^{2}=\gamma_{3} \gamma_{1}^{2}$ from (5-1). The element $\alpha$ detects the element

$$
\sigma\left(t_{1}^{3} t_{2}^{2} t_{3}^{2}\right) \in\left[\mathbb{Z} / 2 \otimes_{\mathcal{A}}\left(P^{\otimes 3}\right)^{\Sigma_{3}}\right]
$$

of [8, Theorem 1.2], under the pairing of a vector space with its dual:

$$
\left[\left(\left(\mathbb{Z} / 2 \otimes_{\Sigma} \Gamma\right)^{\mathcal{A}}\right)_{3, *}\right] \otimes\left[\mathbb{Z} / 2 \otimes_{\mathcal{A}}\left(P^{\otimes 3}\right)^{\Sigma_{3}}\right] \rightarrow \mathbb{Z} / 2 .
$$

We can generate more elements in $\left(\left(\mathbb{Z} / 2 \otimes_{\Sigma} \Gamma\right)^{\mathcal{A}}\right)_{3, *}$ by operating repeatedly upon $\alpha$ with the bigraded Steenrod square $\mathbb{S} q^{0}$. Using (4-3) we find:

$$
\left(\mathbb{S} q^{0}\right)^{q} \alpha=\gamma_{2^{q+2}-1} \gamma_{2^{q}+2^{q+1}-1}^{2}+\gamma_{2^{q+2}+2^{q+1}-1} \gamma_{2^{q+1}-1}^{2}, \quad q \geq 0 .
$$

Under the pairing (6-3), $\left(\mathbb{S} q^{0}\right)^{q} \alpha$ detects the element

$$
\sigma\left(t_{1}^{2^{q+2}-1} t_{2}^{2^{q}+2^{q+1}-1} t_{3}^{2^{q}+2^{q+1}-1}\right)
$$

of $[8$, Theorem 1.2]. 
One is naturally interested in the result of applying the higher squaring operations to $\alpha$. From (4-3) and the Cartan formula we get:

$$
\mathbb{S} q^{1} \alpha=\left(\mathbb{S} q^{1} \gamma_{3}\right)\left(\mathbb{S} q^{0} \gamma_{2}^{2}\right)+\left(\mathbb{S} q^{1} \gamma_{5}\right)\left(\mathbb{S} q^{0} \gamma_{1}^{2}\right)=\left(\gamma_{3}^{2}\right)\left(\gamma_{5}^{2}\right)+\left(\gamma_{5}^{2}\right)\left(\gamma_{3}^{2}\right)=0 .
$$

However:

$$
\begin{aligned}
\mathbb{S} q^{2} \alpha & =\left(\mathbb{S} q^{0} \gamma_{3}\right)\left(\mathbb{S} q^{2} \gamma_{2}^{2}\right)+\left(\mathbb{S} q^{0} \gamma_{5}\right)\left(S q^{2} \gamma_{1}^{2}\right) \\
& =\left(\mathbb{S} q^{0} \gamma_{3}\right)\left(\mathbb{S} q^{1} \gamma_{2}\right)^{2}+\left(\mathbb{S} q^{0} \gamma_{5}\right)\left(\mathbb{S} q^{1} \gamma_{1}\right)^{2}=\gamma_{7} \gamma_{2}^{4}+\gamma_{11} \gamma_{1}^{4},
\end{aligned}
$$

a nonzero element of $\left(\left(\mathbb{Z} / 2 \otimes_{\Sigma} \Gamma\right)^{\mathcal{A}}\right)_{5,20}$. More generally we find for each $r \geq 1$ :

$$
S q^{2^{r}} \mathbb{S} q^{2^{r-1}} \cdots \mathbb{S} q^{2} \alpha=\gamma_{2^{r+2}-1} \gamma_{2}^{2^{r+1}}+\gamma_{2^{r+2}+2^{r+1}-1} \gamma_{1}^{2^{r+1}} .
$$

More generally still we can calculate the results of applying the "admissible" monomial $\mathbb{S} q^{2^{r}} \mathbb{S} q^{2^{r-1}} \cdots \mathbb{S} q^{2}$ to the element $\left.\left(\mathbb{S} q^{0}\right)^{q} \alpha \in\left(\left(\mathbb{Z} / 2 \otimes_{\Sigma} \Gamma\right)^{\mathcal{A}}\right)_{3, *}\right)$ by using the fact that $\mathbb{S} q^{0}$ lies in the center of $\mathcal{H}$ :

$$
\begin{aligned}
& \mathbb{S} q^{2^{r}} \mathbb{S} q^{2^{r-1}} \cdots \mathbb{S} q^{2}\left(\mathbb{S} q^{0}\right)^{q} \alpha=\left(\mathbb{S} q^{0}\right)^{q} \mathbb{S} q^{2^{r}} \mathbb{S} q^{2^{r-1}} \cdots \mathbb{S} q^{2} \alpha \\
& =\gamma_{2^{r+q+2}-1} \gamma_{2^{q+1}+2^{q}-1}^{2^{r+1}}+\gamma_{2^{r+q+2}+2^{r+q+1}-1} \gamma_{2^{q+1}-1}^{2^{r+1}},
\end{aligned}
$$

a nonzero element of $\left(\left(\mathbb{Z} / 2 \otimes_{\Sigma} \Gamma\right)^{\mathcal{A}}\right)_{\left(2^{r+1}+1,10 \cdot 2^{q+r}\right)}$. If we write

$$
\beta(r, q)=\mathbb{S} q^{2^{r}} \mathbb{S} q^{2^{r-1}} \cdots \mathbb{S} q^{2}\left(\mathbb{S} q^{0}\right)^{q} \alpha
$$

for all $r \geq 1, q \geq 0$, and $\beta(0, q)=\left(\mathbb{S} q^{0}\right)^{q} \alpha$, then the set $\{\beta(r, q) \mid r \geq 0, q \geq 0\}$ is linearly independent in $\left(\mathbb{Z} / 2 \otimes_{\Sigma} \Gamma\right)^{\mathcal{A}}$. Indeed, different pairs $(r, q)$ correspond to different bidegrees.

\section{References}

[1] J F Adams, On the structure and applications of the Steenrod algebra, Comment. Math. Helv. 32 (1958) 180-214 MR0096219

[2] M A Alghamdi, M C Crabb, J R Hubbuck, Representations of the homology of $B V$ and the Steenrod algebra. I, from: "Adams Memorial Symposium on Algebraic Topology, 2 (Manchester, 1990)", London Math. Soc. Lecture Note Ser. 176, Cambridge Univ. Press (1992) 217-234 MR1232208

[3] J M Boardman, Modular representations on the homology of powers of real projective space, from: "Algebraic topology (Oaxtepec, 1991)", Contemp. Math. 146, Amer. Math. Soc. (1993) 49-70 MR1224907 
[4] D P Carlisle, R M W Wood, The boundedness conjecture for the action of the Steenrod algebra on polynomials, from: "Adams Memorial Symposium on Algebraic Topology, 2 (Manchester, 1990)", London Math. Soc. Lecture Note Ser. 176, Cambridge Univ. Press (1992) 203-216 MR1232207

[5] M C Crabb, J R Hubbuck, Representations of the homology of $B V$ and the Steenrod algebra. II, from: "Algebraic topology: new trends in localization and periodicity (Sant Feliu de Guíxols, 1994)”, Progr. Math. 136, Birkhäuser, Basel (1996) 143-154 MR1397726

[6] NH V Hu'ng, F P Peterson, $\mathfrak{U}$-generators for the Dickson algebra, Trans. Amer. Math. Soc. 347 (1995) 4687-4728 MR1316852

[7] A S Janfada, R M W Wood, The hit problem for symmetric polynomials over the Steenrod algebra, Math. Proc. Cambridge Philos. Soc. 133 (2002) 295-303 MR1912402

[8] A S Janfada, R M W Wood, Generating $H^{*}\left(\mathrm{BO}(3), \mathbb{F}_{2}\right)$ as a module over the Steenrod algebra, Math. Proc. Cambridge Philos. Soc. 134 (2003) 239-258 MR1972137

[9] M Kameko, Generators of the cohomology of $B V_{4}$, preprint

[10] M Kameko, Generators of the cohomology of $B V_{3}$, J. Math. Kyoto Univ. 38 (1998) 587-593 MR1661173

[11] A Liulevicius, The factorization of cyclic reduced powers by secondary cohomology operations, Mem. Amer. Math. Soc. No. 42 (1962) 112 MR0182001

[12] J P May, A general algebraic approach to Steenrod operations, from: “The Steenrod Algebra and its Applications (Proc. Conf. to Celebrate NE Steenrod's Sixtieth Birthday, Battelle Memorial Inst., Columbus, Ohio, 1970)", Lecture Notes in Mathematics 168, Springer, Berlin (1970) 153-231 MR0281196

[13] D M Meyer, J H Silverman, Corrigendum to: "Hit polynomials and conjugation in the dual Steenrod algebra” [Math. Proc. Cambridge Philos. Soc. 123 (1998) 531-547] by Silverman, Math. Proc. Cambridge Philos. Soc. 129 (2000) 277-289 MR1765915

[14] R J Milgram, Group representations and the Adams spectral sequence, Pacific J. Math. 41 (1972) 157-182 MR0304463

[15] T N Nam, $\mathfrak{A}$-générateurs génériques pour l'algèbre polynomiale, Adv. Math. 186 (2004) 334-362 MR2073910

[16] F P Peterson, A-generators for certain polynomial algebras, Math. Proc. Cambridge Philos. Soc. 105 (1989) 311-312 MR974987

[17] J H Silverman, Hit polynomials and conjugation in the dual Steenrod algebra, Math. Proc. Cambridge Philos. Soc. 123 (1998) 531-547 MR1607993

[18] J H Silverman, W Singer, On the action of Steenrod squares on polynomial algebras. II, J. Pure Appl. Algebra 98 (1995) 95-103 MR1317001

[19] W M Singer, The transfer in homological algebra, Math. Z. 202 (1989) 493-523 MR1022818 
[20] W M Singer, On the action of Steenrod squares on polynomial algebras, Proc. Amer. Math. Soc. 111 (1991) 577-583 MR1045150

[21] W M Singer, On the algebra of operations for Hopf cohomology, Bull. London Math. Soc. 37 (2005) 627-635 MR2143743

[22] W M Singer, Steenrod squares in spectral sequences, Mathematical Surveys and Monographs 129, Amer. Math. Soc. (2006) MR2245560

[23] N E Steenrod, Cohomology operations, Annals of Math. Studies 50, Princeton University Press (1962) MR0145525 Lectures by NE Steenrod written and revised by D B A Epstein

[24] N Sum, The hit problem for the polynomial algebra of four variables, preprint

[25] R M W Wood, Steenrod squares of polynomials and the Peterson conjecture, Math. Proc. Cambridge Philos. Soc. 105 (1989) 307-309 MR974986

[26] R M W Wood, Problems in the Steenrod algebra, Bull. London Math. Soc. 30 (1998) 449-517 MR1643834

[27] N Yoneda, On the homology theory of modules, J. Fac. Sci. Univ. Tokyo. Sect. I. 7 (1954) 193-227 MR0068832

Department of Mathematics, Fordham University

Bronx, NY 10458, USA

singer@fordham . edu

Received: 25 October 2007 